\title{
Les langues du roi. Le rôle de la langue dans la communication de propagande dynastique à l'époque de Charles IV
}

\section{Václav Žůrek}

\section{(2) OpenEdition}

\section{Journals}

Édition électronique

URL : http://journals.openedition.org/ifha/8045

DOI : $10.4000 /$ ifha. 8045

ISSN : 2198-8943

\section{Éditeur}

IFRA - Institut franco-allemand (sciences historiques et sociales)

\section{Édition imprimée}

Date de publication : 31 décembre 2014

ISSN : 2190-0078

\section{Référence électronique}

Václav Žưrek, «Les langues du roi. Le rôle de la langue dans la communication de propagande dynastique à l'époque de Charles IV », Revue de l'IFHA [En ligne], 6 | 2014, mis en ligne le 31 décembre 2014, consulté le 03 mai 2019. URL : http://journals.openedition.org/ifha/8045 ; DOI : 10.4000/ ifha. 8045

Ce document a été généré automatiquement le 3 mai 2019.

(CIFHA 


\title{
Les langues du roi. Le rôle de la langue dans la communication de propagande dynastique à l'époque de Charles IV
}

\author{
Václav Žůrek
}

1 La situation linguistique des pays de la Couronne de Bohême à l'époque des Luxembourg peut être qualifiée de bilinguisme. En effet, le tchèque et l'allemand coexistaient dans le pays, tout comme à la cour, suite à la vague de colonisation allemande du XIIIe siècle et de l'expansion territoriale sous le roi Jean l'Aveugle (1310-1346), surtout en Silésie et en Lusace - deux principautés culturellement profondément germaniques. La cour du roi de Bohême et empereur Charles IV (1346-1378) reflétait cette situation et son caractère cosmopolite s'accentua encore à partir du moment où Prague devint capitale du roi des Romains (1346), puis de l'Empereur (1355) et centre administratif de l'Empire' ${ }^{1}$.

2 Charles IV lui-même prêtait beaucoup d'attention aux langues tant comme élément d'éducation pratique que comme facteur de communication symbolique. Sa réputation de polyglotte est confirmée par plusieurs témoignages contemporains. Lui-même mentionne ses propres connaissances linguistiques dans un passage fameux de son « autobiographie : "Grâce à Dieu, nous savions parler, comprendre, écrire et lire non seulement la langue de Bohême, mais le français, le lombard, l'allemand et le latin, de telle sorte que nous étions capable d'écrire, lire, parler et comprendre aussi l'une comme l'autre de ces langues $»^{2}$. Le chroniqueur Pierre de Zittau mentionne aussi que Charles IV savait écrire, lire et comprendre quatre langues à côté du tchèque ${ }^{3}$. Cette affirmation, qui fait partie du portrait littéraire du prince élu, est confirmée aussi par l'archevêque de Prague Jean de Jenstein dans son éloge funèbre lors des obsèques de Charles IV en $1378^{4}$. Charles IV mit en valeur l'importance de la connaissance des langues étrangères pour les affaires quotidiennes et politiques de l'Empire, ce dont témoigne le chapitre XXXI de la Bulle d'Or de 1356, qui recommande aux Princes-Électeurs d'éduquer leurs enfants pour des raisons pratiques dans les langues suivantes: "en dehors de l'allemand, qu'ils connaissent naturellement, aussi le latin, l'italien et le slave» ce qui veut dire dans ce contexte le 
tchèque $\mathrm{e}^{5}$. Ces langues étaient en effet les moyens principaux de communication dans l'Empire.

3 En raison d'une politique ambitieuse touchant surtout la représentation dynastique des Luxembourg par le biais d'une production littéraire, l'empereur Charles IV avait un grand besoin de lettrés en qualité de conseillers et d'auteurs capables de rédiger des œuvres historiques, politiques, théologiques ou juridiques. La majorité de la production littéraire et savante à la cour de l'empereur Charles IV était rédigée en latin. Il faut toutefois remarquer que pendant son règne, l'activité des traducteurs des œuvres classiques (surtout théologiques et savantes) en langue vernaculaire, c'est-à-dire en tchèque et en allemand, s'intensifia. Une partie des traducteurs travaillait avec le soutien de la cour ce qui, avec la fondation de l'Université à Prague en 1348, rendit possible pendant la deuxième moitié du XIVe siècle l'essor d'une production en vulgaire. À la diffusion des traductions tchèques des œuvres savantes contribua aussi la fondation du monastère des bénédictins de langue et rite slavons dit "Aux Slaves», dans la Nouvelle Ville de Prague. Le souci d'attirer des érudits à la cour ne se nourrissait pas seulement de l'érudition du souverain, mais surtout du programme monarchique, qui peut être qualifié d'historisme idéologique et politique. L'importance de la littérature historique pour Charles IV est démontrée par le nombre des chroniques rédigé à sa commande ${ }^{6}$.

C'est surtout la Cronica Bohemorum de Přibík Pulkava de Radenín ( $† 1380)$ qui doit attirer notre attention dans le contexte linguistique de l'historiographie médiévale en Bohême ${ }^{7}$. Son auteur étudiait à l'université de Prague et fut plus tard directeur de l'école auprès de l'église Saint-Gilles dans la Vieille Ville de Prague. Dans sa chronique, Přibík Pulkava raconte l'histoire des Tchèques depuis le Déluge et leur arrivée en Bohême jusqu'au temps du roi Jean l'Aveugle, père de Charles IV. La rédaction la plus longue s'achève avec la mort d'Élisabeth, sa mère, en 1330.

5 La méthode du travail de chroniqueur a été bien étudiée 8 . Pulkava avait rassemblé les anciennes chroniques tchèques et composé un texte assez clair pourvu d'une structure narrative simple. L'auteur remit la chronique plusieurs fois sur le métier et il la réécrivit probablement sur les demandes et avec les corrections de l'empereur. Tous les indices concordent à indiquer qu'il travailla sous le contrôle direct de l'empereur ou plutôt des gens de son entourage. Nombreuses sont les preuves justifiant le statut officiel de cette chronique.

6 L'auteur insère un grand nombre des diplômes dans son récit historique, ce qui peut avoir un rapport avec la fondation des archives de la couronne à l'époque de Charles IV ${ }^{9}$. Le texte de la chronique est une compilation de plusieurs œuvres historiques connues, l'incipit/le début de la chronique nous informe qu'elle fut rédigée à la commande de Charles IV, qui fit collecter pour l'auteur des chroniques dans tous les monastères et familles nobles ${ }^{10}$. D'après les instructions de Charles IV, Pulkava rejette tous les passages fabuleux et incertains de ses extraits et rédige ce qui reste pour en faire une chronique vraie ${ }^{11}$.

7 C'est la raison probable expliquant la tradition assez particulière du texte. La chronique existe dans plusieurs rédactions. Marie Bláhová a dénombré six rédactions latines ${ }^{12}$. Les versions n'avaient pas le statut de textes provisoires avant une rédaction finale: au contraire, elles circulaient comme des œuvres achevées -nous connaissons un grand nombre de manuscrits des premières versions, mais un seul exemplaire en revanche de la prétendue sixième et dernière rédaction. Les rédactions se distinguent surtout par les sources utilisées. L'empereur, ou un autre personnage apportaient à Pulkava des 
chroniques dont il ignorait jusque-là l'existence. Celui-ci les intégrait dans la nouvelle version et y ajoutait des faits jusqu'alors inconnus. Cette façon de compléter le texte de la chronique illustre bien l'intention générale de l'œuvre. Quand Charles IV acquit le margraviat de Brandebourg (1373), il fit apporter pour Pulkava une chronique de Brandebourg, afin qu'il mêle son histoire à celle de la Bohême. Cette démarche devait contribuer à former une chronique racontant une histoire commune des pays de la Couronne de Bohême à laquelle appartenait désormais la nouvelle principauté. Les extraits dits Brandenburgica apparaissent dans le manuscrit de la cinquième version, sous forme de notes marginales, puis sont incorporés dans le texte de la version suivante et ultime ${ }^{13}$.

Les notes des copistes dans les manuscrits confirment le statut officiel de la chronique dans la réception médiévale. Dans certains manuscrits latins et tchèques, Charles IV est pris pour l'auteur du texte et Pribik Pulkava est qualifié de traducteur en langue tchèque. Dans la tradition médiévale, la chronique était lue comme une œuvre historique officielle des Luxembourg.

Outre ces signes formels, on rencontre des indices parmi les idées présentées dans le texte qui font écho avec l'idéologie de la cour de Prague. Au moment de la narration de l'époque de saint Venceslas (†935), Pulkava incorpore dans sa chronique la légende de saint Venceslas écrite par l'empereur lui-même ${ }^{14}$. Le choix d'insérer la légende officielle est ainsi en accord avec l'intention du texte de Pulkava : puisqu'elle rapporte de manière abrégée l'histoire des débuts du christianisme en Bohême, souligne le lien avec la Grande Moravie et présente saint Venceslas comme le prince idéal ${ }^{15}$. La théorie de la translation de la couronne royale de la Grande Moravie vers la Bohême, insérée par Pulkava dans son récit à propos de l'année 1086, forme l'un des éléments importants de la stratégie de légitimation des rois de Bohême ${ }^{16}$. La continuité entre le règne de Charles IV et ceux de ses prédécesseurs de la dynastie des Přemyslides constitue l'une des idées fondamentales de la chronique.

10 Nous devons prêter aussi attention à la conception de l'identité slave, maniée par Pulkava au début de son récit. La chronique commence avec l'origo gentis des Tchèques. Pulkava souligne l'origine commune de tous les Slaves, un point qu'il relie avec la confusion des langues dans la tour de Babel ${ }^{17}$. Il est donc clair qu'il observe ces ethnies au prisme des langues parlées et que l'appartenance des Tchèques aux Slaves se rapporte par conséquent à la parenté linguistique ${ }^{18}$.

11 Il faisait cheminer les Slaves par le territoire de la Grèce et de Byzance, puis les faisait pénétrer dans le territoire de plusieurs pays : Bulgarie, Russie, Serbie, Dalmatie, Croatie, Bosnie, Carinthie, Istrie et Carniole ${ }^{19}$. Dans la lignée de la chronique de Dalimil, il place le patriarche Czech en Croatie ${ }^{20}$. Pulkava parle alors de Czech (le mot tchèque pour désigner le «Tchèque ») et n'utilise pas le nom latinisé Bohemus comme le fait Cosmas de Prague ${ }^{21}$.

12 Pulkava met l'accent sur le sentiment d'appartenance slave en reliant l'origine des Tchèques à celle des Polonais et des autres Slaves voisins : dans son récit, il relève leur parenté commune. Il parle ainsi de Lech, frère de Czech et ancêtre des Polonais, qui arrive avec lui et continue à travers les montagnes vers le nord où il s'installe ${ }^{22}$. D'après Pulkava, les gens de la race de Lech allèrent à la génération suivante peupler la Russie, la Poméranie, mais aussi la Moravie, la Misnie, le Brandebourg et la région de Bautzen ou Lusace. Dans ce passage, la chronique de Pulkava accomplit de façon assez démonstrative son programme initial, c'est-à-dire de servir de fondement historiographique et de socle de communication à la politique de Charles IV. Dans le tableau des peuples slaves 
apparentés avec les Tchèques, on peut en effet trouver sans peine la plupart des pays vers lesquels se dirigeait l'expansion de la dynastie des Luxembourg ou bien s'étendait leur domination actuelle. Cette intention explique aussi que le Brandebourg ait été ajouté dans cette énumération sous la forme d'une note marginale dans le manuscrit écrit en 1374, soit à l'époque où les Luxembourg l'incorporèrent parmi les pays composant leur patrimoine.

Pulkava connut un succès énorme et son influence fut renforcée par la traduction du latin en tchèque, ce qui, bien sûr, élargit le nombre des lecteurs potentiels. Plus tard, probablement pendant le XVe siècle, la chronique fut même traduite deux fois en allemand, dans la deuxième langue vernaculaire en usage dans les pays de la Couronne de Bohême. Ces deux traductions allemandes ne peuvent être rattachées au milieu curial, seul leur aspect linguistique permet de les localiser en périphérie dialectique. Le texte fut traduit en deux dialectes allemands régionaux différents - l'allemand de Silésie d'une part et celui de Bavière de l'autre ${ }^{23}$. Il s'en est conservé plus de 40 manuscrits jusqu'à nos jours, dont 21 latin, 16 tchèques et deux allemands ${ }^{24}$.

Bien que les traductions des œuvres latines en tchèque eussent été soutenues par la cour et que cette chronique de Pulkava fût un texte quasi officiel, il n'est pas facile de déterminer le milieu d'origine de la traduction. On peut constater que le traducteur tchèque a suivi l'original de façon assez fidèle, tantôt il a raccourci le récit, tantôt il l'a étoffé. Il a même traduit des passages relatifs à l'histoire du Brandebourg et une partie des diplômes insérés. Or les fautes dans la conversion de la datation latine montrent que le traducteur ne peut être identique à l'auteur. La traduction tchèque est antérieure aux années 1390, une datation permise grâce aux fragments du plus ancien manuscrit ${ }^{25}$.

Or, on risque de trop schématiser si l'on veut attribuer tous les mérites de l'essor de la littérature en langue tchèque au seul souverain Charles IV et à son proche entourage. Malgré le soutien actif apporté à l'activité de traduction en langue vernaculaire, qui constituait l'un des axes de la politique culturelle de la cour de Charles IV, il paraît évident que la langue tchèque était, dans l'idéologie de la cour, réservée principalement aux œuvres dotées d'un haut statut symbolique, surtout théologique et didactique (bible, patristique, légende, vocabulaire etc.). La langue tchèque n'était pas par exemple utilisée sous Charles IV dans l'administration du royaume de Bohême. Tandis que le nombre des diplômes latins et allemands est à peu près le même et que Charles a même fait dresser quelques chartes en français, nous ne lui connaissons aucune charte en langue tchèque ${ }^{26}$.

En ce qui concerne les textes rédigés en latin dans l'entourage de l'empereur, et traduits plus tard en vernaculaire, tout porte à croire que la demande de traductions vernaculaires provenait des lecteurs potentiels suffisamment intéressés par les textes pour en financer la traduction. Au temps de leur rédaction, ces œuvres n'étaient probablement pas pensées dans la perspective d'une version vernaculaire. À côté de Pulkava et de la légende de saint Venceslas, on peut mentionner aussi l'autobiographie de Charles IV, son ordo du sacre, les Moralitates et le code légal Maiestas Carolina. Les trois premières œuvres furent traduites aussi en allemand.

17 Charles IV insista beaucoup, dans sa politique intérieure sur la coexistence des Tchèques et des Allemands au sein du royaume. Les chroniqueurs à la cour de Charles IV (François de Prague, Beneš Krabice de Weitmile et Přibík Pulkava), quand ils ont repris les récits des chroniques de Dalimil et de Pierre de Zittau, en ont ôtées intentionnellement toutes les mentions de conflits ethniques entre Tchèques et Allemands, afin de concorder avec l'une 
des conceptions de l'empereur, attaché à conserver dans son royaume une coexistence sans conflit ${ }^{27}$.

Le prologue de la version tchèque de la chronique de Pulkava confirme l'idée, suivant laquelle la traduction a pris naissance dans un autre milieu que celui de son origine. Il reprend de façon libre quelques informations de l'incipit latin mentionné et répète que la chronique fut écrite à la commande de Charles IV, qui fit collecter des chroniques dans tous les monastères et que dans cette chronique « toutes les choses fabuleuses et fausses sont abandonnées et que ce qui est certain et vrai y est déposé ${ }^{28}$. Or, il continue de façon assez différente et suit plutôt une intention patriotique surprenante. Le traducteur insiste sur la valeur de l'intérêt de son pays et rejette celles des affaires étrangères.

dois apprendre les faits de ton pays sans chercher les nouvelles des autres pays ni les merveilles quelconques. (...) Or, les sages disent que si quelqu'un veut voir ses (proches) en bonne santé, il doit demander des nouvelles de son pays sans veiller à la cuisine italienne ${ }^{29}$ ».

Dans l'esprit de ses mécènes, le traducteur formule dans le prologue l'idée d'une préférence pour l'histoire et les affaires du pays au détriment de l'étranger, une conception contradictoire avec le caractère cosmopolite de la cour de Charles IV et les idées universalistes de son règne. Tout porte à croire qu'il est nécessaire de situer cette traduction dans un autre contexte que celui de son origine. Bien que le récit de la chronique latine de Pulkava se concentre surtout sur l'histoire de la Bohême, nous n'y trouvons pas de formulation d'une telle nature.

21 Le mécène de la traduction tchèque de Pulkava doit donc être recherché parmi les nobles de la cour de Venceslas IV. La noblesse tchèque s'efforçait alors d'imiter le souverain, surtout Charles IV, et en même temps de rivaliser avec son fils Venceslas, et ils ont trouvé un moyen d'asseoir leur position vis-à-vis du roi actuel dans le soutien accordé à la littérature tchèque. Ils se sont appropriés un rôle originairement royal mais, paradoxalement, ont ainsi contribué à la propagation des idées fondamentales du programme de légitimation dynastique des Luxembourg formulées à l'époque de Charles IV. Ce sont les manuscrits latins et surtout tchèques, avec les textes de la cour de Charles IV très répandus dans les bibliothèques du XVe siècle, qui ont aidé à imposer la vision de l'histoire de la Bohême présentée par Přibík Pulkava.

La noblesse de la fin de XIVe siècle s'est identifiée avec la vision de l'histoire formulée dans l'entourage de Charles IV, qui influença alors la conscience historique jusqu'à la fin du Moyen Âge. La décision de traduire en langue vernaculaire des textes importants pour la diffusion des idées liées à la légitimité dynastique de Luxembourg a donc en Bohême contribué à la formation d'une identité partagée des habitants des pays de la Couronne de Bohême. Cette narration, surtout sous la forme que lui confère une chronique de Pulkava, attachée à raconter une histoire commune, contribua à la formulation d'un des piliers de la nation tchèque au début du XVe siècle ${ }^{30}$. 


\section{NOTES}

1. Sur la vie et le règne de Charles IV, voir par Ferdinand Seibt, Karl IV. Ein Kaiser in Europa 1346-1378, München : Süddeutscher Verlag, 1978 ; Jiří Spěváček, Karel IV. Život a dílo (1316-1378), Praha : Svoboda, 1980.

2. Pierre Monnet/Jean-Claude Schmitt (éd.), Vie de Charles IV de Luxembourg, Paris: Les Belles Lettres, 2010, p. 56-57.

3. «Chronicon Aulae Regiae » in : Josef Emler (éd.), Fontes rerum Bohemicarum (désormais FRB) IV, Praha: Spolek historický, 1884, p. 318 : «Quadruplex ipse scit lingwagium, Gallicum, Lombardicum, Teutunicum et Latinum ; in hiis lingwis scit scribere, legere et intelligere, et se optime potest expedire ".

4. Sermo factus per dominum Johannem archiepiscopum Pragensem post mortem imperatoris Caroli IV, in : Josef Emler (éd.), FRB III, Praha : Spolek historický, 1882, p. 427.

5. Wolfgang D. Fritz (éd.), Bulla aurea Karoli IV. imperatoris anno MCCCLVI promulgata (=MGH Fontes iuris XI), Weimar : Herrmann Böhlaus Nachfolger, 1972, p. 90.

6. Cf. Marie Bláhová, «Offizielle Geschichtsschreibung in dem mittelalterlichen böhmischen Ländern ", in: Jaroslaw Wenta (dir.), Die Geschichtsschreibung in Mitteleuropa. Projekte und Forschungsprobleme, Toruń : Wydawnictwo Uniwersytetu Nikołaja Kopernika, 1999, p. 24-40.

7. «Przibiconis de Radenin dicti Pulkavae Chronicon Bohemiae », in : Josef Emler/Jan Gebauer (éd.), FRB V, Praha : Spolek historický, 1893, p. 1-326.

8. Franz Palacky, Würdigung der alten böhmischen Geschichtsschreiber, Praha: A. Borrosch, 1830, p. 173-192 ; Marie Bláhová, Kroniky doby Karla IV., Praha : Svoboda, 1987, p. 572-580.

9. Ivan Hlaváček, "L'exploitation du matériel diplomatique dans les chroniques de la Bohême médiévale », in : Jean-Philippe Genet (dir.), L’Historiographie médiévale en Europe, Paris : Éditions CNRS, p. 84-86.

10. «Przibiconis de Radenin dicti Pulkavae Chronicon Bohemiae», op. cit., p. 207 : « ad mandatum serenissimi ac invictissimi principis et domini, domini Karoli quarti, divina favente clemencia Romanorum imperatoris ac Boemie regis ".

11. Ibid., p. 207.

12. M. Bláhová, Kroniky doby Karla IV., op. cit., p. 573-576.

13. Ulrike Hohensee, «Solus Woldemarus sine herede mansit superestes. Brandenburgische Geschichte in der Sicht Pulkawas » in: Turbata per aequora mundi. Dankesgabe an Eckhard MüllerMertens, Hannover : Hahnsche Buchhandlung, 2001, p. 115-129.

14. Anton Blaschka (éd.), Die St. Wenzelslegende Kaiser Karls IV. Einleitung, Text, Kommentar, Praha : Verlag der Deutschen Gesellschaft der Wissenschaften und Künste für die Tschechoslowakische Republik, 1934.

15. « Przibiconis de Radenin dicti Pulkavae Chronicon Bohemiae », op. cit., p. 18-22.

16. Ibid., p. 54. Cf. Marie Bláhová, « ... kako jest koruna z Moravy vyšla... ('Translatio regni' ve Staročeské kronice tzv. Dalimila.) », Mediaevalia Historica Bohemica, nº 3, 1993, p. 165-176.

17. Ibid, p. 4.

18. D'après Fr. Graus, les chroniqueurs à la cour de Charles IV sont les premiers à traiter le sujet de l'origine slave des Tchèques. Voir František Graus, Nationenbildung der Westslaven im Mittelalter, Sigmaringen : Thorbecke, 1980, p. 130-132.

19. «Przibiconis de Radenin dicti Pulkavae Chronicon Bohemiae », op. cit., p. 4.

20. Ibid. 
21. À propos de Bohemus et de ses transformations dans l'historiographie voir František Graus, Lebendige Vergangenheit. Überlieferung im Mittelalter und in den Vorstellungen vom Mittelalter, Cologne : Böhlau, 1975, p. 89-92.

22. «Przibiconis de Radenin dicti Pulkavae Chronicon Bohemiae », op.cit., p. 5.

23. Vlastimil Brom, "Aus der offiziellen böhmischen Historiographie Karls IV. Die PulkavaChronik in drei Sprachversionen ", Brünner Beiträge zur Germanistik und Nordistik, $\mathrm{n}^{\circ}$ 15, 2010, p. 5-19. Vlastimil Brom a aussi préparé aussi l'édition électronique parallèle des textes de la Chronique de Pulkava et de la Vita Karoli en latin, en tchèque et en allemand et elle est accessible sur le lien: https://www.phil.muni.cz/german/projekty/hmb/. Pour la version allemande, il s'agit de la seule édition existante.

24. M. Bláhová, Kroniky doby Karla IV., op. cit., p. 577-579, énumère et décrit les manuscrits.

25. Ibid.

26. Ivan Hlaváček, «Dreisprachigkeit im Bereich der Böhmischen Krone. Zum Phänomen der Sprachbenutzung im böhmischen diplomatischen Material bis zur hussitischen Revolution », in : Anna Adamska/Marco Mostert (dir.), The Development of Literate Mentalities in East Central Europe, Turnhout : Brepols, 2004, p. 289-310.

27. Martin Nodl, «Nationalismus und Nationalbewusstsein zu Beginn des 14. Jahrhunderts und Karls Bemühen um ein konfliktfreies Bild des Zusammenlebens von Tschechen und Deutschen in Böhmen » in : Stefan Albrecht (dir.), Die Königsaaler Chronik. Eine Bestandsaufnahme, Frankfurt a. M.: Peter Lang, 2012, p. 198-240.

28. «Przibiconis de Radenin dicti Pulkavae Chronicon Bohemiae », op. cit., p. 211.

29. Ibid., La métaphore de la «cuisine italienne» est unique en vieux tchèque et doit probablement signifier la culture étrangère.

30. Cette recherche a bénéficié du financement du Conseil européen de la recherche au titre du Programme de la Communauté européenne, septième programme-cadre (FP7/2007-2013)/ERC conventions de subvention $n^{\circ} 263672$.

\section{AUTEUR}

\section{VÁCLAV ŽŮREK}

(Centre d'études médiévales, Prague) 